\title{
Characterization of Low-Frequency Noise of MOSFETs Using 2-D Device Simulator
}

\author{
Hyunchul Nah, Young June Park, and Hong-Shick Min \\ School of EECS, Seoul National University, Seoul, Korea \\ woods@amity.snu.ac.kr \\ Chanho Lee \\ School of EE, Soongsil University, Seoul, Korea \\ Hyungsoon Shin \\ School of IEE, Ewha Womans University, Seoul, Korea
}

\begin{abstract}
Low-frequency noise of the MOSFET was simulated using the transfer impedance method together with a 2-D device simulator. In the simulation, various generation-recombination (GR) components such as SRH recombination centers and surface traps of various lifetimes are taken into account. It is shown that lifetimes of traps determine the characteristics of lowfrequency noise. Also is shown that the GR process not only at the surface but also in the bulk may be responsible for the low-frequency noise.
\end{abstract}

\section{Introduction}

As the MOSFET becomes an important active device in the RF applications, the prediction of the noise characteristics is a challenging issue. Recently, a noise simulation algorithm using the PDE based 2-D device simulator has been developed [1]. It is based on the transfer impedance method [2], in which the Langevin type noise sources for both diffusion and GR noise are considered as the fundamental forcing terms of the fluctuation. In the previous papers, the simulation results of the high-frequency noise of the MOSFET [1] and the GR noise of simple resistors [3] have been reported

The low-frequency noise, however, has been rarely mentioned although it has been known to be the major cause of the various phase noises in the oscillators or mixers. Very often the low-frequency noise spectra of devices can be explained in terms of various GR noise components. It is known that the low-frequency noise characteristics can be properly predicted if all the fluctuation mechanisms of the carrier population in a device are clarified.

In this paper, GR noise sources with various lifetimes and locations in the device are considered in the framework of transfer impedance method implemented in the SNU-2D [4] to predict the comprehensive characteristics of the low-frequency noise of MOSFET devices. 


\section{Theory}

The physical model considered as a basis for the noise analysis is the bipolar driftdiffusion model and the trap continuity equation together with the appropriate boundary conditions [1];

$$
\begin{aligned}
& \nabla^{2} \varphi+\frac{q}{\varepsilon}(p-n+C)+\frac{\rho_{t}}{\varepsilon}=0 \\
& \frac{\partial n}{\partial t}-\frac{1}{q} \nabla \bullet \mathbf{J}_{n}+R_{n}-G_{n}=\gamma_{n} \\
& \frac{\partial p}{\partial t}+\frac{1}{q} \nabla \bullet \mathbf{J}_{p}+R_{p}-G_{p}=\gamma_{p} \\
& \frac{\partial n_{t}}{\partial t}=R_{n}-G_{n}-R_{p}+G_{p}+\gamma_{t},
\end{aligned}
$$

where the symbols are given their usual meanings and $n_{t}$ and $\gamma$ s indicate the electron density in trap states and the corresponding Langevin forcing terms, respectively.

The last equation can be back-substituted into the first three equations using the relations between $n_{t}$ and $\rho_{t}, R_{n}, G_{n}, R_{p}$, and $G_{p}$ [5]. With these equations (the Poisson, and electron and hole continuity equation), the noise formulae can be established by the transfer impedance method [1][3] to obtain the Green function describing the coupling between the distributed noise sources and the device terminals. The fluctuation sources in the resulting equations are transformed to

$$
\tilde{s}_{\varphi}=\Gamma_{\varphi t} \tilde{\gamma}_{t}, \quad \tilde{s}_{n}=\tilde{\gamma}_{n}+\Gamma_{n t} \tilde{\gamma}_{t}, \quad \text { and } \quad \tilde{s}_{p}=\tilde{\gamma}_{p}+\Gamma_{p t} \tilde{\gamma}_{t},
$$

where subscript $\varphi, n$, and $p$ represent the Poisson and electron and hole continuity equation. The expressions for the $\Gamma$ 's can be found in [3].

In the consideration of the multi-trap, we have assumed that the traps are independent to other traps, i.e. there are no direct carrier transitions between traps. To consider the silicon-oxide interface where the very fast (dominant in DC) and the very slow (believed to be the cause of $1 / f$ noise) traps coexist, the interface states with different surface recombination velocities has been included.

The cross power spectral density between the open circuit voltage $\delta v_{i}$ and $\delta v_{j}$ at terminal $i$ and $j$ is given by [1][3]

$$
S_{\delta v_{i}, \delta v_{j}}=\sum_{\alpha, \beta=\varphi, n, p} \int_{\Omega} \widetilde{G}_{\alpha}\left(\mathbf{r}_{i}, \mathbf{r}, \omega\right) K_{\tilde{s}_{\alpha}, \tilde{s}_{\beta}}(\mathbf{r}, \omega) \widetilde{G}_{\beta}^{*}\left(\mathbf{r}_{j}, \mathbf{r}, \omega\right) d \mathbf{r}
$$

where $G$ and $K$ are the scalar transfer impedance and the microscopic local noise source, respectively.

The microscopic local noise source $(K)$ can be obtained from the equations for $s_{\varphi}, s_{n}$, and $s_{p}[3]$ whose expressions are not repeated here.

The correlation matrix of the noise current power spectrum can be obtained from the calculated correlation matrix of the noise voltage power spectrum and the AC admittance matrix $\mathbf{Y}$ as: 


$$
\mathbf{S}_{\mathbf{I}}=\mathbf{Y} \mathbf{S}_{\mathbf{V}} \mathbf{Y}^{\dagger}
$$

where $\mathbf{Y}^{\dagger}$ is the complex conjugate of $\mathbf{Y}$.

\section{Simulation results and discussions}

Using the fore-mentioned scheme, an n-channel MOSFET was simulated. Its structure has $0.8 \mu \mathrm{m}$ gate length with $200 \AA$ gate oxide thickness. The threshold voltage of the device is about $0.3 \mathrm{~V}$.

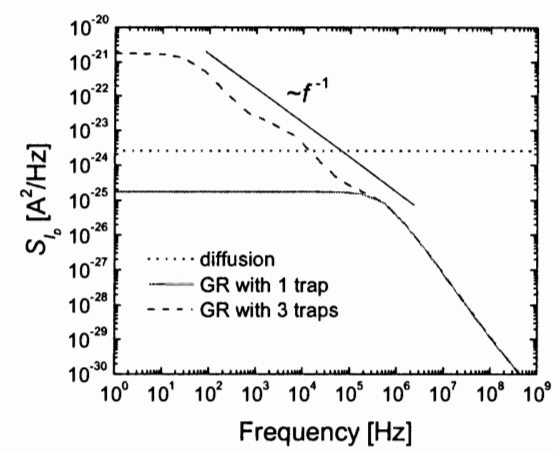

Fig. 1. The simulated noise spectrum of n-channel MOSFET at $V_{D}=V_{G}=3 \mathrm{~V}$. The solid line is from one-trap with $\tau_{n}=\tau_{p}=10^{-7} \mathrm{~s}$. The dashed line is three-traps with $10^{-7} \mathrm{~s}, 10^{-5} \mathrm{~s}$ and $10^{-3} \mathrm{~s}$

Fig. 1 shows the simulated power spectrum of the drain noise current $\left(S_{i d}\right)$ at $V_{G}=$ $V_{D}=3 \mathrm{~V}$. The solid line represents the GR noise due to the single trap whose lifetimes for electron and hole and the trap density are $10^{-7} \mathrm{~s}$ and $10^{11} \mathrm{~cm}^{-3}$, respectively. Generally, there exist traps with various lifetimes in the device. Usually, the only fastest trap is taken into account when the DC characteristics of the device is to be obtained, because it dominates the GR rate. For the AC and noise behaviour, however, the slower traps can be no longer ignored. The dashed line in the figure shows the data including three kind of traps whose lifetimes are $10^{-3} \mathrm{~s}$, $10^{-5} \mathrm{~s}$ and $10^{-7} \mathrm{~s}$. The spectrum can be considered as the sum of three Lorenzians due to each traps and the envelope roughly looks like $1 / f$ spectrum as expected. It can be seen that the corner frequency which is an important parameter of $1 / f$ noise can properly predicted by the careful decision of the trap parameters.

In the above figure, no surface recombination has been considered; only bulk recombination gives the $1 / f$ spectrum. Apparently, there are rare probability for very slow traps (ex. trap of lifetime of $1 \mathrm{~s}$ ) to exist in bulk [6]. So the measured $1 / f$ spectrum having $1 / f$ characteristics at very low frequency may be the sum of bulk GR noise (intermediate frequency) and the surface GR noise (very low frequency). The bias dependence of $1 / f$ noise is shown in Fig. 2. Though the accurate level of the noise spectrum cannot be predicted, the bias dependency of the simulated results are reasonable. Many experiments report that in the strong inversion and linear region, the $1 / f$ spectrum is almost constant along with the gate bias (left) [7]. The 
right figure of Fig. 2 is the $V_{D}$ dependence of the $1 / f$ noise. $S_{I D} / I_{D}{ }^{2}$ is expected to be nearly flat in the linear region, increase in between the linear and saturation region and become flat again in the saturation region [8]. The simulated result basically follows the trend of the measurement but the point at which the spectrum flattens rather deviates from the saturation bias point, whose reason is the topic of the further work.
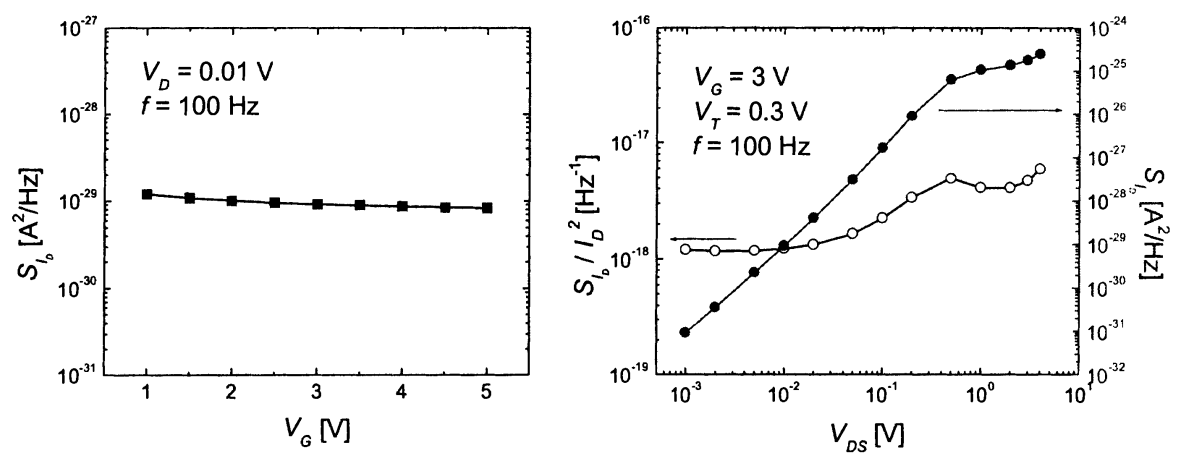

Fig. 2. $V_{G}$ (left) and $V_{D}$ (right) dependence of $1 / f$ noise.

\section{Acknowledgement}

This work was supported by the National Research Laboratory Project of the Ministry of Science and Technology, a Collaborative Project for Excellence in Basic System IC Technology, and Brain Korea 21 Project.

\section{References}

[1] F. Bonani, G. Ghione, M. R. Pinto and R. K. Smith, IEEE Trans. Electron Devices, vol. 45 , no. 1 , p. 261,1998

[2] C. M. van Vliet, IEEE Trans. Electron Devices, vol. 41, no. 11, p. 1902, 1994

[3] F. Bonani and G. Ghione, Solid-State Electronics, vol. 43, p. 285, 1999

[4] W. S. Choi, J. K. Ahn, Y. J. Park, H. S. Min and C. G. Hwang, IEEE Trans. CAD, vol. 13, p. 899,1994

[5] A. Gnudi, P. Ciampolini, R. Guerrieri, M. Rudan and G Baccarani, Proceeding of NASCODE V, p. 207,1987

[6] A. van der Ziel, Noise in Solid State Devices and Circuits, John Wiley and Sons, 1986

[7] C. H. Park and Y. J. Park, Solid-State Electronics, vol. 44, p. 2053, 2000

[8] C. H. Park, H. S. Min and Y. J. Park, Proceedings of ICNF 2001, to be published 